\title{
Dental anomalies in first-degree relatives of transposed canine probands
}

\author{
Adriana Bartolo ${ }^{1}$, Neville Calleja ${ }^{2}$, Fraser McDonald ${ }^{3}$ and Simon Camilleri ${ }^{3}$ \\ The aim of this study was to investigate and compare the inheritance pattern and prevalence of inheritable dental anomalies in a sample \\ of patients with maxillary canine-first premolar transposition and their first-degree relatives with a sample of palatally displaced \\ canine families. Thirty-five consecutive maxillary canine-first premolar transposition probands and 111 first-degree relatives were \\ matched to 35 consecutive palatally displaced canine probands and 115 first-degree relatives. These were assessed for palatally \\ displaced canines and incisor-premolar hypodontia. Parental age at birth of the proband was also noted. The results revealed that \\ (i) there is no difference in the overall prevalence of palatally displaced canine or incisor-premolar hypodontia between the groups of \\ relatives; (ii) first-degree relatives of bilateral palatally displaced canine probands have a higher prevalence of palatally displaced \\ canine and incisor-premolar hypodontia than those with unilateral palatally displaced canine; and (iii) maternal age at birth of the \\ maxillary canine-first premolar transposition probands was significantly higher than that of the palatally displaced canine probands. \\ The results suggest that maxillary canine-first premolar transposition and palatally displaced canine are unlikely to be different \\ genetic entities and also indicate environmental or epigenetic influences on dental development. \\ International Journal of Oral Science (2015) 7, 169-173; doi:10.1038/ijos.2014.72; published 30 January 2015
}

Keywords: ectopic; epigenomics; genetics; hypodontia; orthodontics; tooth eruption

\section{INTRODUCTION}

Palatally displaced canine (PDC) is a complication of dental development. It frequently occurs in conjunction with familial incisor-premolar hypodontia (IPH), being related, inheritable, dental anomalies $\left(\right.$ OMIM 189490) ${ }^{1-4}$ (Figure 1a). The precise aetiology of PDC and of IPH is unknown. Evidence exists for the influence of a major autosomal dominant gene in both conditions. ${ }^{5-6}$ However, the incomplete penetrance and variable expression is consistent with a polygenic model overlaid by environmental factors. ${ }^{7}$ Discordance of monozygotic twins ${ }^{6,8}$ suggests that environmental or epigenetic factors may influence dental development; indeed, low birth weight has been implicated in the aetiology of hypodontia. ${ }^{9}$

Tooth transposition is a rare event, with a mean prevalence of $0.33 \% .{ }^{10}$ It is defined as an exchange of position between two adjacent teeth including their roots, or the development and eruption of a tooth in a position normally occupied by another non-adjacent tooth ${ }^{11}$ (Figure 1b). The commonest type of transposition is maxillary canine/first premolar transposition (MxCP1). Frequent association of tooth transpositions with other inheritable dental anomalies such as IPH and PDC ${ }^{11-12}$ in affected individuals suggests a shared genetic aetiology for these anomalies.

Based on the patterns of anomalies in affected individuals, the suggestion has been put forward that MxCP1 and PDC are different entities, associated with variants in the MSX1 and PAX9 genes. ${ }^{13}$ Furthermore, several studies have associated an increased prevalence
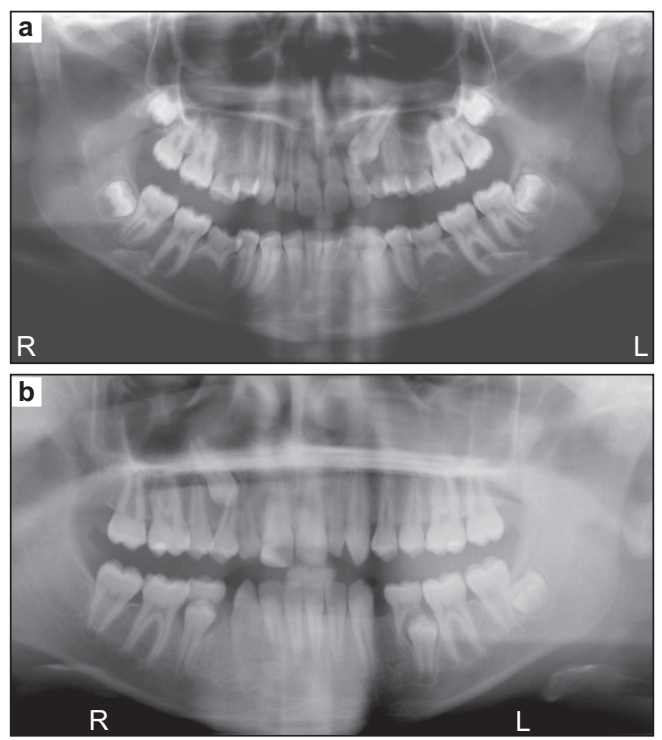

Figure 1 The two varieties of ectopic maxillary canine under study. (a) A palatally displaced canine can be seen on the left, together with developmentally missing second premolars and retained deciduous teeth. (b) A maxillary caninefirst premolar transposition is on the right, together with developmentally missing upper lateral incisors and lower first premolars and three third molars.

${ }^{1}$ Broadstreet Dental Centre, Hamrun, Malta; ${ }^{2}$ Department of Public Health, Medical School, University of Malta, Tal-Qroqq, Malta and ${ }^{3}$ Dental Institute, Department of Orthodontics, Kings College London, London, UK

Correspondence: Dr S Camilleri, Dental Institute, Department of Orthodontics, Kings College London, Tower Wing, Great Maze Pond, London SE19RT, UK

E-mail: simon.camilleri@kcl.ac.uk

Accepted 29 October 2014 
of hypodontia with the occurrence of MxCP1 in individuals. ${ }^{11,14-15}$ Evidence against these associations has been brought forward, ${ }^{12,16}$ but given the dissimilarities between the phenotypes, it is possible that the genotypes may be different and analysis of the pattern of transmission within families may shed light on this matter. Family studies on PDC probands have demonstrated an association between PDC and hypodontia ${ }^{3,17}$ and an association between PDC and maxillary canine transpositions. ${ }^{6}$ However, no family studies involving $\mathrm{MxCP} 1$ probands have been published to our knowledge.

The aim of the study was to investigate the prevalence of palatally displaced canines and of IPH in a sample of probands with MxCP1 and their first-degree relatives as compared to a sample of PDC probands and their first-degree relatives.

\section{MATERIALS AND METHODS}

Ethical approval for the study was granted by the University of Malta Research Ethics Committee reference no. 44/2009.

\section{Sample selection}

A pilot study indicated that the incidence of IPH in the MxCP1 sample to be $15 \%$ and $3 \%$ in $\mathrm{MxCP} 1$ and $\mathrm{PDC}$ relatives, respectively. A sample size of 88 in each group was required in order to detect a difference with a power of $80 \%$, at $5 \%$ significance.

\section{Participant selection}

All MxCP1 probands under treatment or review were identified from the records at the Orthodontic Clinic of the Dental Surgery Department, Mater Dei Hospital, Malta and a private practice in Sliema, Malta and invited to attend for a dental examination, together with their first-degree family members. Similarly, a sample of consecutive probands with a PDC and their first-degree family members were also asked to take part in the study. Informed written consent was obtained in all cases prior to the examination. Individuals who did not attend the dental examination or who refused consent were excluded. Inclusion criteria for the MxCP1 and PDC groups were (i) probands with $\mathrm{MxCP} 1$ and their first-degree family members; and (ii) probands who were Caucasian with Maltese citizenship. Exclusion criteria were (i) probands and first-degree family members with syndromes and/or medical conditions that may affect the eruption or formation of teeth; (ii) probands with other types of canine transposition or displacement; and (iii) individuals aged less than 9 years, as not all permanent tooth germs may have shown radiographic evidence of calcification.

Thirty-five MxCP1 probands met the selection criteria and together with 111 first-degree relatives formed the MxCP1 group. Similarly, 35 PDC probands and 115 first-degree family members formed the PDC group. In all, a total of 296 patients attended for a dental examination.

\section{Examination}

This was carried out by the same examiner (AB). Reproducibility was assessed by re-examining 20 first-degree family members and their radiographs, 4 weeks after the first examination. The dentition was assessed visually, using a mouth mirror and operating light. A dental history was elicited and all available records were examined. If a history could not be ascertained from the patient, permission was obtained to request the records from their general dental practitioner. Heritable dental anomalies were recorded and categorized as (i) ectopic teeth and (ii) developmentally missing teeth; excluding third molars. Diagnosis was by clinical and radiographic examination. The published local values for EC and IPH were used for comparison. ${ }^{6,18}$
During the assimilation of data, the impression of a difference in the ages of the parents of the probands of the groups was formed. The maternal and paternal ages at birth of probands were compared between groups and compared to the national average.

\section{Statistical analysis}

The results were processed using Stata/IC for Windows release 11.2 statistical analysis software (StataCorp LC, College Station, TX, USA) and Microsoft Excel 2010 spreadsheet software (Microsoft Corporation, Redmond, WA, USA). Intra-examiner reliability was analysed using the kappa statistic. Summary statistics were first compiled using all individuals. Subsequently, the probands were removed from the study and PDC groups and the prevalence rates of PDCs and IPH compared between first-degree relatives of both groups. Data from relatives of bilateral cases were compared to those of unilateral cases. Familial relative risk was calculated as $\left(\lambda_{\mathrm{R}}=\kappa_{\mathrm{R}} / \kappa\right)$ where $\kappa$ is the population prevalence and $\kappa_{\mathrm{R}}$ is the percentage of relatives affected. Statistical analysis treated each family member as being independent using Fisher's exact test and Pearson Chi-squared tests. Further analysis with hierarchical logistic regression using Intercooled STATA 11.2 showed that the clustering effect within families was not statistically significant and therefore, no such bias was introduced in our results. The parental ages were compared using the unpaired Student's $t$-test and a Bonferroni correction applied for multiple testing.

\section{RESULTS}

\section{Summary statistics}

Reproducibility was 95\% for all inheritable dental anomalies (kappa value $=0.857$ ). There were a total of 166 female and 130 male participants in the study. Pearson chi-squared tests showed that there was no significant difference in the sizes or gender distribution of the groups. Out of the 35 probands of the MxCP1 group, there was a higher prevalence of females than males $(60 \%)$, with five bilateral cases. Out of 35 PDC probands, 70\% were females and there were nine bilateral cases. Twelve MxCP1 probands and five PDC probands had IPH; a Pearson Chi-squared test found the difference between the groups to be significant $(P=0.05)$. There was no difference in the overall prevalence of IPH and PDC combined $(P=0.27)$.

\section{Analysis of first-degree relatives}

There were 111 first-degree relatives in the MxCP1 group and 115 first-degree relatives in the PDC group, 226 individuals in all. The inheritable dental anomalies were analysed in the first-degree relatives of the MxCP1 and PDC groups. Of the subjects selected from the MxCP1 group, 24 participants (22\%) had an inheritable dental anomaly. Out of the PDC group, 27 participants (24\%) had an inheritable dental anomaly. A Pearson Chi-squared test did not find the difference between the groups to be significant $(P=0.877)$. There were 20 affected female and four affected male relatives in the MxCP1 group, a ratio of 5:1 (F:M). The PDC group had 16 affected female relatives and 11 affected males, a ratio of 1.45:1 (F:M). A Fisher Exact test did not find the difference between the groups to be significant $(P=0.073)$.

Out of the MxCP1 group, 15 participants (14\%) had PDCs. Out of the PDC group, there were 22 participants (19\%) with PDCs. Both groups had a higher prevalence of PDCs than the general population, $P=0.003$ for the MxCP1 group and $P>0.001$ for the PDC group. The number of first-degree relatives with PDCs was higher in the PDC group than the MxCP1 group, but a Pearson Chi-squared test did not find the difference between the groups to be statistically significant 


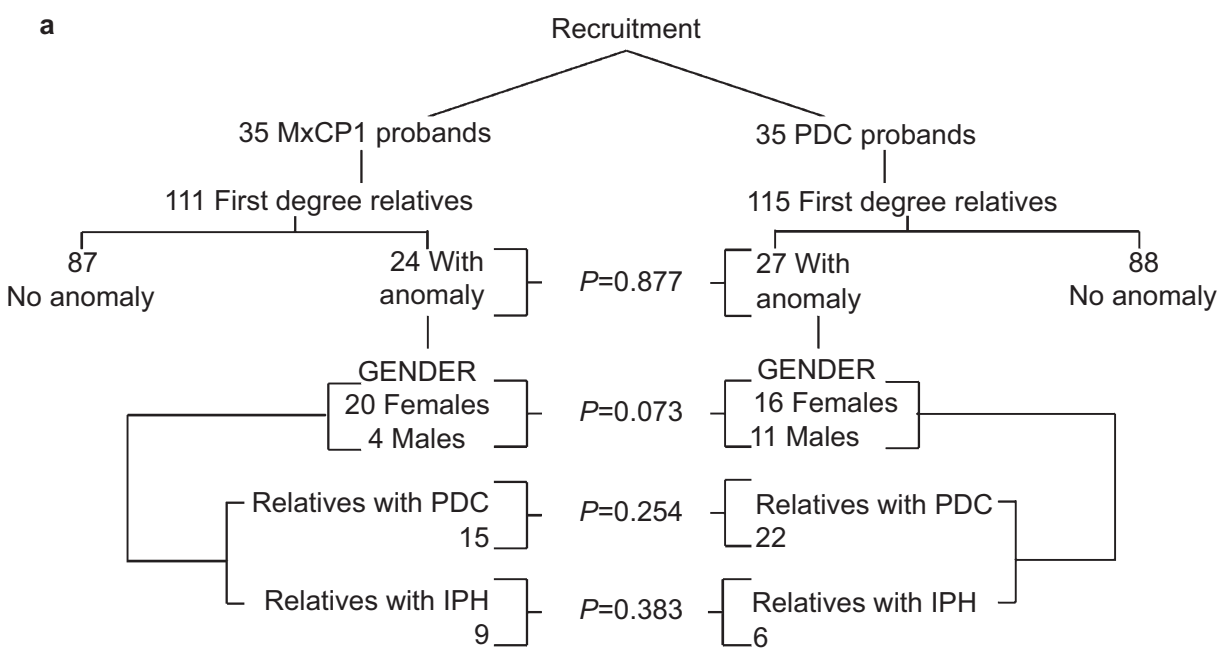

b
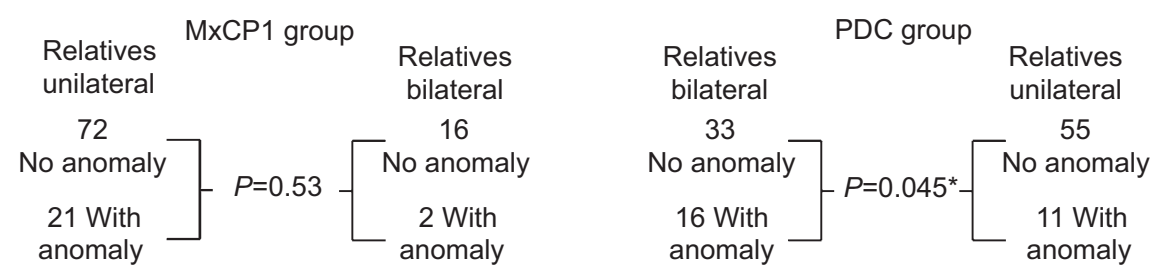

Figure 2 Diagrammatic representation of the comparison of the first-degree relatives of the MxCP1 and PDC groups. (a) There is no difference in the distribution of gender or in the prevalence or distribution of PDC and IPH between the groups. (b) Comparison of all anomalies between unilateral and bilateral cases of MxCP1 and PDC. There is a significant difference in the PDC group but not in the MxCP1 group. IPH, incisor-premolar hypodontia; MxCP, maxillary canine/first premolar transposition; PDC, palatally displaced canine.

( $P=0.254$ ). The relative risk for PDC in MxCP1 relatives was 2.5 and that for PDC relatives was 3.6.

Out of the $\mathrm{MxCP} 1$ group, there were nine (8\%) participants with IPH. Out of the PDC group, there were six (5\%) participants with IPH. A Fisher Exact test found the figure for IPH to be higher than that of the general population for the MxCP1 group $(P<0.001)$ and the PDC group $(P=0.002)$. The number of first-degree relatives with IPH was higher in the MxCP1 group than the PDC group, but a Pearson Chisquared test did not find the difference between the groups to be statistically significant $(P=0.383)$ (Figure $2 \mathrm{a})$. The relative risk for IPH was 2.53 for the MxCP1 group and 1.63 for the PDC group. There were no cases of any relatives of either group with transposed teeth of any sort.

\section{Comparison of unilateral and bilateral PDC and MxCP1 cases}

In this study, there were 66 first-degree relatives of probands with unilateral PDC, out of which 11 (17\%) had a dental anomaly. There were 49 first-degree relatives of probands with bilateral PDC, out of which $16(33 \%)$ had a dental anomaly. A Pearson Chi-squared test found this difference to be statistically significant $(P=0.045)$. Ten of the bilateral PDC group relatives had a PDC and so the relative risk for PDC in the bilateral PDC subgroup was 5.94. Three had IPH and the relative risk for IPH was 1.91. There were 93 first-degree relatives of probands with unilateral MxCP1, out of which 21 (23\%) individuals had a dental anomaly. There were 18 first-degree relatives of probands with bilateral MxCP1, out of which two (11.1\%) individuals had a dental anomaly. A Fisher Exact test did not find this difference to be statistically significant $(P=0.53)$ (Figure $2 \mathrm{~b})$.

\section{Parental age at delivery of probands}

The mean age of the mother at delivery was statistically higher for MxCP1 probands (32.18 years) than PDC probands (28.09 years) $(P=0.005$; 95\% CI: $1.29-6.79)$. Following application of a Bonferroni correction, the result remained statistically significant. The population mean for mother's age at delivery is 26 years (National Statistics Office, Malta). There was no significant difference for the father's age and the population mean for father's age was not available. Three out of the four probands affected bilaterally in this sample were all born of mothers 38 years of age or over.

\section{DISCUSSION}

In this study, the phenotypes of the probands were similar to that of other studies into transposed and ectopic canines, with a female predilection and with a similar distribution and a higher prevalence of dental anomalies than the general population. There was a significant increase in the prevalence of IPH noted between the MxCP1 probands over the PDC probands in this group. This supports previous reports associating MxCP1 with hypodontia.

There was a marked difference in the gender of affected relatives, with 3.6 times the number of affected females in the MxCP1 as compared to the PDC group. However, the difference was not statistically significant, possibly due to the small numbers. Any further investigation will have to await the incorporation of more $\mathrm{MxCP} 1$ cases.

Both groups of first-degree relatives had a higher prevalence of PDC and IPH than the general population. However, there was no difference in the number of relatives affected with either of the two anomalies, pointing to a similar penetrance in both groups. This is in 
contrast to the finding of an increase in IPH in the $\mathrm{MxCP} 1$ probands, as one would expect a consequential increase in the prevalence of IPH in close relatives. As the number of affected relatives is the same, the evidence here suggests a similar association with IPH for both groups. The pattern of tooth agenesis in all the subjects under study was that of familial IPH, with no more than six missing teeth, excluding third molars. Even allowing for the variable expression often seen, mutations in MSX1 or PAX9 would be expected to produce a more severe pattern of molar/premolar agenesis in relatives.

Bilateral PDC cases had significantly higher numbers of affected relatives than unilateral $\mathrm{PDC}$ cases, suggesting greater genetic penetrance. It is, however, difficult to separate environmental from genetic influences. The higher number of relatives could also mean some environmental factor predisposing to the disruption of dental development, as shared environmental factors are customary in families. There was, surprisingly, no similar increase for bilateral MxCP1 cases.

There were no relatives in either group affected with MxCP1 or transpositions of any sort. This in itself is not unexpected in a study this size, given the rarity of the event. However, it is notable that there are very few reports of familial occurrence of $\mathrm{MxCP} 1$ and none where parent-child transmission is demonstrated. ${ }^{11,19-22}$ An unexpected finding was the association of $\mathrm{MxCP} 1$ with increased maternal age, with a mean difference of 4.09 years between the groups. The maternal age after which the risk of severe developmental defects increases sharply is taken to be 35 years; however, increasing maternal age is a continuum rather than a threshold effect ${ }^{23}$ and the 4 -year difference may be biologically significant in this respect. The elevated maternal age of the bilateral MxCP1 probands may reflect this continuum. Increased maternal age is associated with a number of non-chromosomal developmental defects ${ }^{24}$ and alteration of methylation patterns of developmental genes, ${ }^{25}$ supporting the possibility of intrauterine environmental factors affecting dental development. Permanent teeth develop from the successional lamina, which forms from the fifth month in utero till the tenth month of age; ${ }^{26}$ therefore, events impacting on developmental genes within this time range may very well affect the dentition.

Although published evidence points to the action of a dominant major gene or genes in the aetiology of $\mathrm{PDC},{ }^{6}$ the variable expression and incomplete penetrance seen in relatives suggests the additional influence of a number of minor genes and possibly also environmental or epigenetic effects on gene transcription to modify the phenotype. This is in keeping with a polygenic model and it is possible that maternal age acts as an aggravating factor, where a genetic predisposition exists, to produce the MxCP1 phenotype. This may also help explain the dearth of reports of parental transmission, the disparity in IPH prevalence between the probands and relatives and the difference in penetrance between bilateral PDC and MxCP1 cases as if development of $\mathrm{MxCP} 1$ were not directly due to variations in the DNA nucleotide sequence; it is unlikely that transmission would take place. This is an interesting avenue for further study.

From a clinical aspect, the relative risk quantifies the chances of finding a dental anomaly in close relatives and highlights the necessity of examining siblings of affected children. First-degree relatives of $\mathrm{MxCP} 1$ and PDC probands have a much higher risk of PDC or IPH than the general population; however, the highest risk group are the relatives of bilateral PDC cases, where the chances of developing PDC is nearly six times the population prevalence in this study.

The main limitation of the study is the sample size, constrained by the rarity of MxCP1 and hence, the difficulty in identifying a large number of cooperative families. The general population from which this sample was drawn is small, with genetic characteristics of a small, young population and therefore, the results may not be strictly applicable to larger, more established populations. On the other hand, the published literature on tooth transpositions consists chiefly of case reports, or collections of mixed transpositions. Where the phenotype under study has been limited to one type, most are a mix of populations. This sample is limited to one transposition phenotype and one population. The relative genetic homogeneity shields to some extent against population stratification with any genetic effects more likely to be shared between individuals than in larger populations.

\section{CONCLUSION}

This is the first reported family study investigating and comparing the phenotypic dental features in a sample of MxCP1 and PDC individuals and their first-degree relatives. The prevalence and distribution of dental anomalies between the two groups of relatives was similar, pointing to a similar genetic aetiology. The most penetrant subgroup was the bilateral PDC group and these families should be considered as high risk for dental anomalies. Increased maternal age may be a factor in the development of MxCP1.

\section{ACKNOWLEDGEMENTS}

This study was funded under the Joint King's College London/University of Malta agreement.

1 Bjerklin K, Kurol J, Valentin J. Ectopic eruption of maxillary first permanent molars and association with other tooth and developmental disturbances. Eur J Orthod 1992; 14(5): 369-375.

2 Peck S, Peck L, Kataja M. The palatally displaced canine as a dental anomaly of genetic origin. Angle Orthod 1994; 64(4): 249-256.

3 Pirinen S, Arte S, Apajalahti S. Palatal displacement of canine is genetic and related to congenital absence of teeth. J Dent Res 1996; 75(10): 1742-1746.

4 Baccetti T. A controlled study of associated dental anomalies. Angle Orthod 1998; 68(3): 267-274.

5 Arte S, Nieminen P, Apajalahti S et al. Characteristics of incisor-premolar hypodontia in families. J Dent Res 2001; 80(5): 1445-1450.

6 Camilleri S, Lewis CM, McDonald F. Ectopic maxillary canines: segregation analysis and a twin study. J Dent Res 2008; 87(6): 580-583.

7 Brook $\mathrm{AH}$. A unifying aetiological explanation for anomalies of human tooth number and size. Arch Oral Biol 1984; 29(5): 373-378.

8 Townsend G, Hughes T, Luciano M et al. Genetic and environmental influences on human dental variation: a critical evaluation of studies involving twins. Arch Oral Biol 2009; 54(Suppl 1): S45-S51.

9 Keene HJ. Birth weight and congenital absence of teeth in twins. Acta Genet Med Gemellol (Roma) 1971; 20(1): 23-42.

10 Papadopoulos MA, Chatzoudi M, Kaklamanos EG. Prevalence of tooth transposition. A meta-analysis. Angle Orthod 2010; 80(2): 275-285.

11 Peck L, Peck S, Attia Y. Maxillary canine-first premolar transposition, associated dental anomalies and genetic basis. Angle Orthod 1993; 63(2): 99-109; discussion 110.

12 Camilleri S. Maxillary canine anomalies and tooth agenesis. Eur J Orthod 2005, 27(5): 450-456.

13 Peck S, Peck L, Kataja M. Concomitant occurrence of canine malposition and tooth agenesis: evidence of orofacial genetic fields. Am J Orthod Dentofacial Orthop 2002 122(6): 657-660.

14 Plunkett DJ, Dysart PS, Kardos TB et al. A study of transposed canines in a sample of orthodontic patients. Br J Orthod 1998; 25(3): 203-208.

15 Cho SY, Chu V, Ki Y. A retrospective study on 69 cases of maxillary tooth transposition. J Oral Sci 2012; 54(2): 197-203.

16 Ely NJ, Sherriff M, Cobourne MT. Dental transposition as a disorder of genetic origin. Eur J Orthod 2006; 28(2): 145-151.

17 Svinhufvud E, Myllärniemi S, Norio R. Dominant inheritance of tooth malpositions and their association to hypodontia. Clin Genet 1988; 34(6): 373-381.

18 Camilleri S, Mulligan K. The prevalence of malocclusion in Maltese schoolchildren as measured by the Index of Orthodontic Treatment Need. Malta Med J 2007; 19(1): 19_ 24.

19 Allen WA. Bilateral transposition of teeth in two brothers. Br Dent J 1967; 123(9): 439-440.

20 Feichtinger C, Rossiwall B, Wunderer H. Canine transposition as autosomal recessive trait in an inbred kindred. J Dent Res 1977; 56(12): 1449-1452. 
21 Newman GV. Transposition: orthodontic treatment. J Am Dent Assoc 1977; 94(3): 544-547.

22 Segura JJ, Hattab F, Ríos V. Maxillary canine transpositions in two brothers and one sister: associated dental anomalies and genetic basis. ASDC J Dent Child 2002 69(1): 54-58, 12

23 Cleary-Goldman J, Malone FD, Vidaver J et al. Impact of maternal age on obstetric outcome. Obstet Gynecol 2005; 105(5 Pt 1): 983-990.

24 Reefhuis J, Honein MA. Maternal age and non-chromosomal birth defects, Atlanta1968-2000: teenager or thirty-something, who is at risk? Birth Defects Res Part A Clin Mol Teratol 2004; 70(9): 572-579.

25 Adkins RM, Thomas F, Tylavsky FA et al. Parental ages and levels of DNA methylation in the newborn are correlated. BMC Med Genet 2011; 12: 47.
26 Orban B, Bhaskar SN. Orban's oral histology and embryology. New Delhi: Elsevier India, 2011.

This work is licensed under a Creative Commons Attribution-

(c) (i) $\Theta$ NonCommercial-NoDerivs 3.0 Unported License. The images or other third party material in this article are included in the article's Creative Commons license, unless indicated otherwise in the credit line; if the material is not included under the Creative Commons license, users will need to obtain permission from the license holder to reproduce the material. To view a copy of this license, visit http://creativecommons.org/licenses/ by-nc-nd/3.0/ 